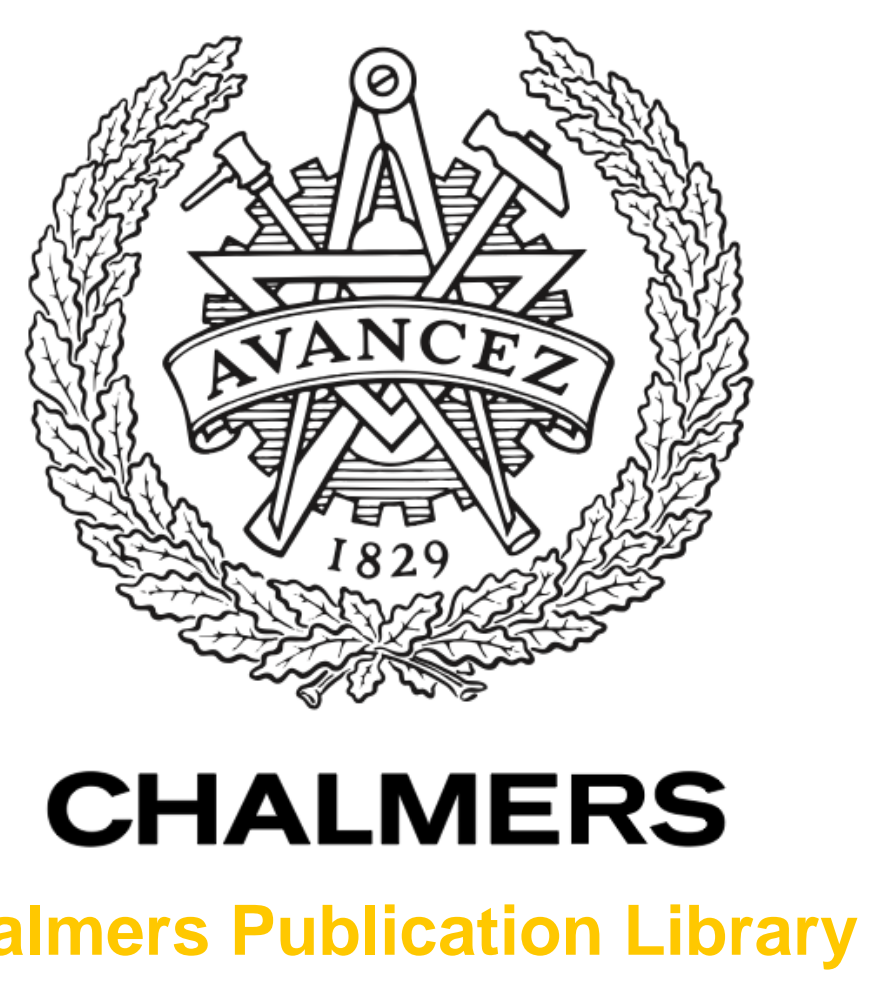

Chalmers Publication Library

Superconducting subterahertz fast nanoswitch

This document has been downloaded from Chalmers Publication Library (CPL). It is the author's version of a work that was accepted for publication in:

Jetp Letters (ISSN: 0021-3640)

Citation for the published paper:

Kuzmin, L. ; Tarasov, M. ; Otto, E. (2007) "Superconducting subterahertz fast nanoswitch". Jetp Letters, vol. 86(4), pp. 275-277.

http://dx.doi.org/10.1134/S002136400716013810.1

$134 / \mathrm{s} 0021364007160138$

Downloaded from: http://publications.lib.chalmers.se/publication/82330

Notice: Changes introduced as a result of publishing processes such as copy-editing and formatting may not be reflected in this document. For a definitive version of this work, please refer to the published source. Please note that access to the published version might require a subscription.

Chalmers Publication Library (CPL) offers the possibility of retrieving research publications produced at Chalmers University of Technology. It covers all types of publications: articles, dissertations, licentiate theses, masters theses, conference papers, reports etc. Since 2006 it is the official tool for Chalmers official publication statistics. To ensure that Chalmers research results are disseminated as widely as possible, an Open Access Policy has been adopted.

The CPL service is administrated and maintained by Chalmers Library. 


\title{
Superconducting Subterahertz Fast Nanoswitch
}

\author{
L. Kuzmin ${ }^{a}$, M. Tarasov ${ }^{b}$ É. Otto ${ }^{a}$, A. Kalabukhov ${ }^{a}$, G. Yassin ${ }^{c}$, \\ P. Grimes ${ }^{c}$, and P. Mauskopf ${ }^{d}$ \\ ${ }^{a}$ Chalmers University of Technology, Göteborg SE41296, Sweden \\ ${ }^{b}$ Institute of Radio Engineering and Electronics, Russian Academy of Sciences, \\ ul. Mokhovaya 11/7, Moscow, 125009 Russia \\ e-mail: tarasov@hitech.cplire.ru \\ ${ }^{c}$ Oxford University, Oxford OX1 3RH, United Kingdom \\ ${ }^{d}$ Cardiff University, Cardiff CF24 3YB, United Kingdom \\ Received May 31, 2007; in final form, June 26, 2007
}

\begin{abstract}
A superconducting thin-film nanoswitch for the subterahertz frequency range has been proposed, developed, fabricated, and tested. The switch makes it possible to modulate the microwave signal or switch it between two branches of a circuit with low losses and high speed. The switch can be naturally integrated with superconducting high-sensitive detectors. Its application makes it possible to avoid the use of massive slow mechanical modulators and to improve the measurement accuracy in decisive astrophysical experiments such as the investigation of the anisotropy of the cosmic microwave background.
\end{abstract}

PACS numbers: 74.45.+c, 74.50.+r

DOI: $10.1134 / \mathrm{S} 0021364007160138$

Many measurements in the subterahertz frequency range are restricted by the capabilities of waveguide mechanical switchers and modulators. A switching speed of no higher than tens of Hertz, direct losses of about several decibels, switching instability, the large sizes of devices, and the impossibility of their integration with cryogenic detectors are among such restrictions. Investigation of the polarization of the cosmic microwave background (relic radiation) can be mentioned among the critical measurements. The ground, balloon, and space projects Clover [1], EBEX [2], BICEP [3], etc., in a frequency range of $40-450 \mathrm{GHz}$ are under development now. The components of the anisotropy of the radiation are measured in a polarimeter with the switching of the phase difference between the channels, $0 / 90^{\circ}$ and $0 / 180^{\circ}$. The application of both mechanical and magnetic switches based on the Faraday effect in ferrite rods cannot provide the required measurement accuracy up to $10^{-4}$. The application of pin diodes also does not ensure small losses at these frequencies. At the same time, it seems to be very attractive to use superconducting structures to reduce losses and to obtain compatibility with superconducting detectors, bolometers, and mixers.

Let us consider an electromagnetic wave propagating along a planar transmission line across which a superconducting nanobridge is connected. A required switch must transmit a signal without losses in the open state and completely reflect it in the closed state. This property is ensured if the impedance of the switching element is much larger than the impedance of the line in the open state and is much smaller than the impedance of the line in the closed state. The impedance of the superconducting nanobridge in the closed state, $Z_{\text {off }}$, is the sum of the geometric inductance $L_{\mathrm{g}}$ and kinetic inductance $L_{\mathrm{k}}$ of the superconducting current

$$
Z_{\text {off }}=i \omega\left(L_{\mathrm{g}}+L_{\mathrm{k}}\right) \text {. }
$$

The impedance in the open state, $Z_{\text {on }}$, includes normal resistance $R_{\mathrm{n}}$, but does not include kinetic inductance:

$$
Z_{\text {on }}=R_{\mathrm{n}}+i \omega L_{\mathrm{g}} .
$$

As a result, the normal resistance of a strip must prevail. The material and geometry must obviously be chosen so as to maximally ensure the inequality $\omega L_{\mathrm{g}} \ll R_{\mathrm{n}}$. For a niobium nitride strip $5 \mu \mathrm{m}$ in length, $500 \mathrm{~nm}$ in width, and $20 \mathrm{~nm}$ in thickness with a resistivity of $200 \mu \Omega \mathrm{cm}$, the resistance of the bridge is equal to $1 \mathrm{k} \Omega$ and the geometric inductance is $3 \mathrm{pH}$.

The kinetic inductance of a thin film can be calculated by the formula $L_{\mathrm{k}}=\mu_{0} \lambda^{2}(l / w t)$, where $\mu_{0}=1.25 \times$ $10^{-6} \mathrm{H} / \mathrm{m}$ is the magnetic constant; $\lambda=200 \mathrm{~nm}$ is the London penetration depth; and $l, w$, and $t$ are the strip length, width, and thickness, respectively. The kinetic inductance of our strip is equal to $13 \mathrm{pH}$. As a result, the total inductive resistance at a frequency of $225 \mathrm{GHz}$ is equal to $22 \Omega$ and partial transmission occurs in the closed state of the line with $Z_{0}=70 \Omega$ and the mismatch factor $K_{p}=4 R_{0} R_{s} /\left(R_{0}+R_{s}\right)^{2}=0.7$. In the case of the full height waveguide or the line with an impedance of $300 \Omega$, the mismatch factor is equal to 0.25 in the 


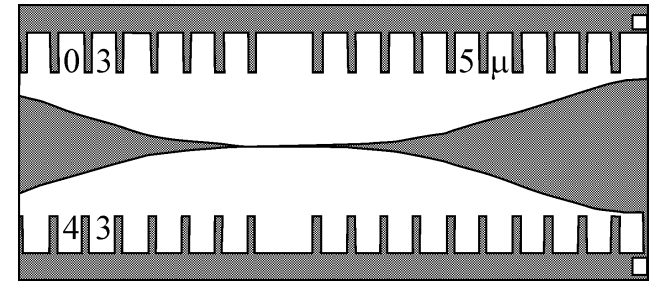

Fig. 1. Optical microscope image of the sample with a finline, which has a length of $5 \mu \mathrm{m}$ and a width of $0.3 \mu \mathrm{m}$, and smooth transitions to the main-section waveguide. The structure number (43) is indicated on the left.

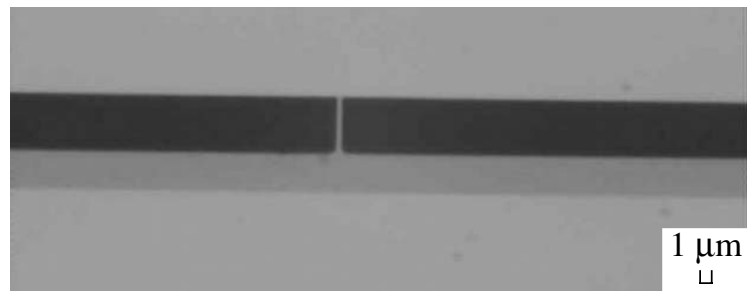

Fig. 2. Image of the $\mathrm{NbN}$ nanobridge located at the center of the slotline.

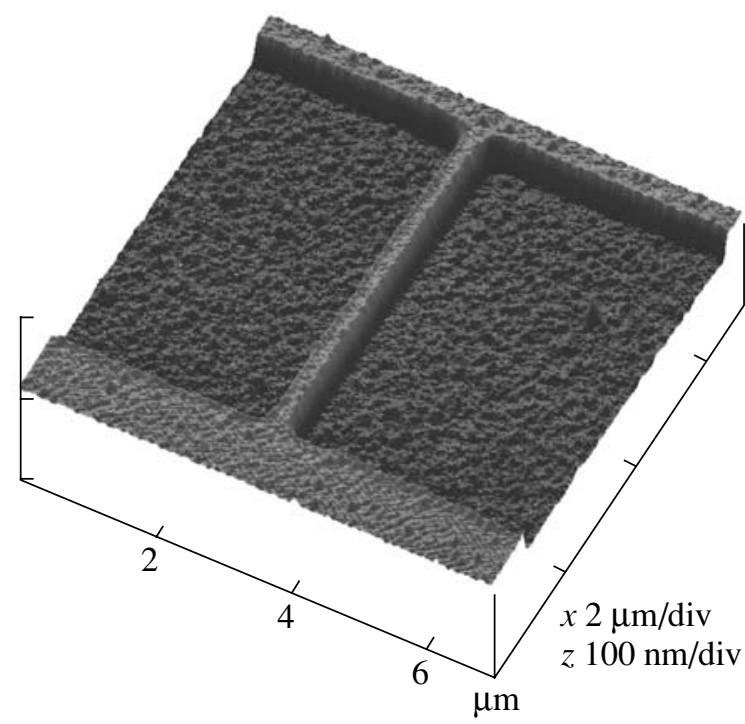

Fig. 3. Atomic-force microscope image of the bridge with a length of $5 \mu \mathrm{m}$, a width of $0.4 \mu \mathrm{m}$, and a thickness of $20 \mathrm{~nm}$.

closed state. However, the mismatch factor in the open state is equal to 0.7 ; i.e., the direct losses of a signal are equal to $70 \%$.

Capacitive matching can be used in order to increase attenuation in the closed state. In this case, the nanobridge is connected to the transmission line through capacitors forming a resonance tank circuit with the inductances of the bridge. The impedance in the closed state

$$
Z_{\text {off }}=i \omega\left(L_{\mathrm{g}}+L_{\mathrm{k}}\right)+2 / i \omega C=\left(2-\omega^{2} L C\right) / i \omega C
$$

at the resonance frequency can be close to zero and be responsible for total reflection. In our case, capacitances at a frequency of $225 \mathrm{GHz}$ must be equal to $65 \mathrm{fF}$. The matching band at a level of $-15 \mathrm{~dB}$ is equal to $50 \mathrm{GHz}$. The switch operation band with resonant capacitors is determined by the quality factor of the resonance tank circuit and can reach $30 \%$.

In order to extend the matching band and to simplify the design, the strip can be shortened to $1 \mu \mathrm{m}$. In this case, inductances decrease by a factor of 5 and acceptable modulation depth values can be obtained even without the use of matching capacitances. One can also use a material with higher resistivity (e.g., high-temperature superconductor) and a line with higher impedance. It is convenient to use the following simple relation for the ratio of the impedances in the open and closed states

$$
Z_{\text {on }} / Z_{\text {off }}=\rho / \omega \mu_{0} \lambda^{2},
$$

which is independent of the strip geometry.

We also point to the small power consumption of such a switch type, $P=V^{2} / R_{\mathrm{n}}$. In this case, the dissipation power is equal to $2 \mu \mathrm{W}$ and can be easily reduced by reducing the bridge volume.

As a test structure, we used a slotline with $Z_{0}=70 \Omega$ with smooth transitions to the total height of the main section waveguide, a so-called finline (see Fig. 1). The structure was formed through electron lithography by the ion etching method on a quartz substrate with a thickness of $200 \mu \mathrm{m}$. The niobium nitride film was deposited by the magnetron sputtering method of the niobium cathode on a heated substrate in a plasma in the argon-nitrogen mixture. Then, the bilayer photoresist was deposited and the pattern of the electrodes and slotline was formed. After development and cleaning, a 50 -nm gold film with chromium sublayer for better adhesion was deposited by thermal sputtering. Then, the resist is lifted off. The next stage is electron lithography for the formation of the niobium nitride nanobridge. Then, reactive ion etching was performed in the $\mathrm{CF}_{4}$ plasma. As a result, a narrow long bridge was formed (see Fig. 2), whose detailed structure is seen in the atomic force microscope image (see Fig. 3).

After fabrication, the samples were dc tested at liquid helium temperature. Figures 4 and 5 show the current-voltage characteristics and switching characteristics, respectively. Below $100 \mathrm{kHz}$, we observe only a small delay at a level of about $0.5 \mu \mathrm{s}$, which is associated with the measuring circuit time constant.

Then, the wafers are cut by a high-speed diamond saw to the size corresponding to the groove size in the waveguide unit with the waveguide $0.55 \times 1.1 \mathrm{~mm}$ in cross section. An OB-65 backward-wave oscillator was used as a radiation source for measurements near $230 \mathrm{GHz}$. We manufactured a cryogenic insert with a circular oversized waveguide, where the waveguide unit with a nanoswitch and a detector based on a high- 


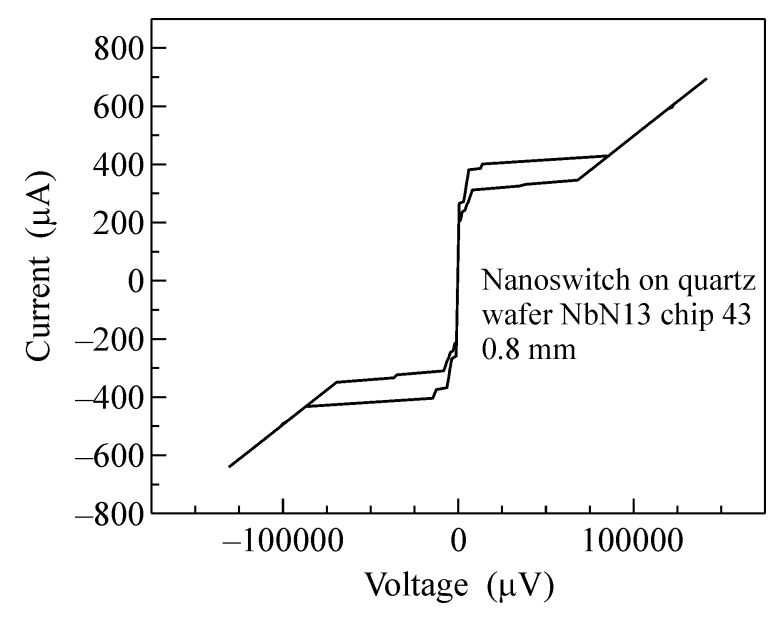

Fig. 4. Current-voltage characteristic of the bridge with a width of $800 \mathrm{~nm}$, a resistance of $200 \Omega$, and a critical current of $300 \mu \mathrm{A}$.

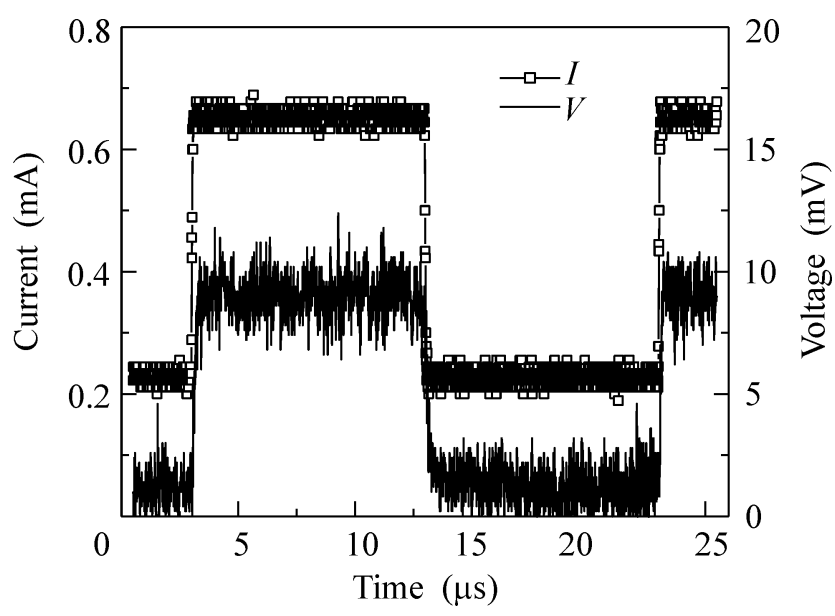

Fig. 5. Switching characteristics of the bridge. Lines $I$ and $V$ are the time dependences of a bias current and the measured voltage, respectively.

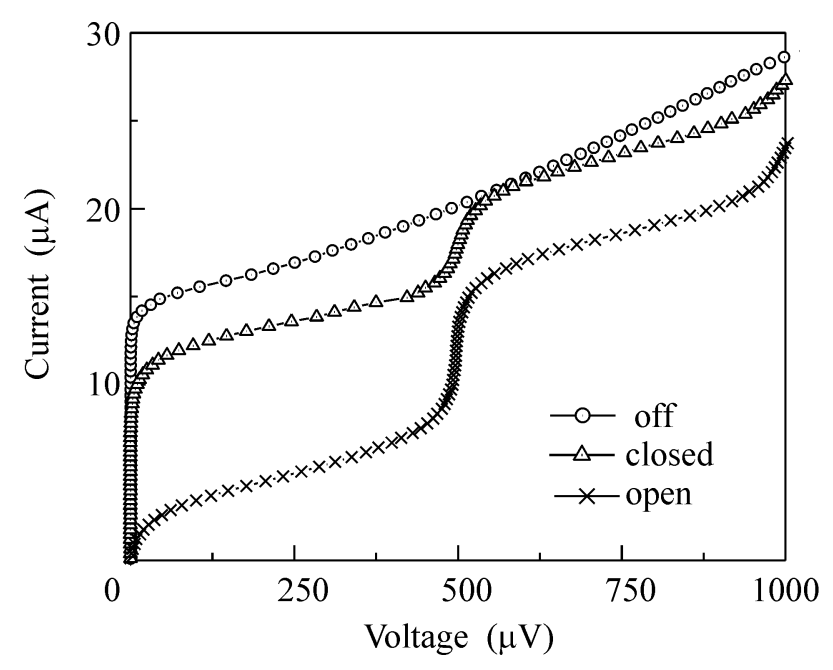

Fig. 6. Current-voltage characteristics of the Josephson detector. The lines marked as open, closed, and off are for an open key, closed key, and turned-off radiation source. temperature Josephson junction were located. The measurement of the current-voltage characteristic of the Josephson junction allows the determination of the signal level at the output of our switch. Figure 6 shows the current-voltage characteristics in the absence of a signal, with the turned-on generator and closed and open keys. It is seen that a small transmission of the signal occurs in the closed position and the level in the open position increases by a factor of 5 , i.e., by $14 \mathrm{~dB}$.

The resulting modulation depth is somewhat lower than that expected for the completely matched switch $(100 \%)$, but it is higher than that for a simple superconducting strip without resonance tuning (30\%). In our waveguide design, capacitors formed by the edges of the slotline entering into a groove in the waveguide (nonemitting slot) severe as tuning capacitors. The corresponding capacitance is determined as $C=\varepsilon_{0} \varepsilon l w / t=$ $4 \times 8.85 \times 10^{-12} \times 4 \times 10^{-3}=140 \mathrm{fF}$ in terms of the relative permittivity of the wafer, $\varepsilon=4$, length $l=4 \mathrm{~mm}$, width $w=0.2 \mathrm{~mm}$, and thickness $t=0.2 \mathrm{~mm}$. In order to satisfy the requirements for a particular switch, it can be shortened with an increase in the resistance (by a decrease in thickness); or the impedance of the line can be increased; or integrated on chip capacitors can be used; or other materials, including high-temperature superconductors, can be used. Note that the limiting speed of such a switch can be comparable with an estimate of less than 36 ps or up to $16 \mathrm{GHz}$ made for a similar topology in an $\mathrm{NbN}$ hot-electron bolometer [4].

A new fast planar subterahertz superconducting switch based on a niobium nitride nanobridge has been proposed, developed, produced, and investigated. The switching characteristics have been measured at frequencies up to $100 \mathrm{kHz}$. The modulation depth in the waveguide mount at a frequency of $230 \mathrm{GHz}$ is larger than $14 \mathrm{~dB}$. In order to satisfy the requirements for a particular experiment, the characteristics can be improved by using additional resonant capacitors or superconductors with higher normal-state resistivity.

This study was supported by the Swedish Science Agency, the Russian Foundation for Basic Research (project no. 05-02-19650 NTsNIL-a), and FANI (project no. 02.513.11.3157).

\section{REFERENCES}

1. G. Yassin, in Proceedings of 15th International Symposium on Space Terahertz Technology (Northampton, Mass., 2004).

2. P. Oxley, Proc. SPIE 5543, 320 (2004).

3. B. G. Keating, Proc. SPIE 4843, 284 (2003).

4. K. S. Il'in, M. Lindgren, M. Currie, et al., Appl. Phys. Lett. 76, 2752 (2000). 